\title{
Detection of Phenotype Modifier Genes Using Two-Locus Linkage Analysis in Complex Disorders Such as Major Psychosis
}

\author{
Alexandre Bureau $^{\mathrm{a}, \mathrm{b}}$ Jordie Croteau ${ }^{\mathrm{a}}$ Chantal Mérette $^{\mathrm{a}, \mathrm{c}}$ Alain Fournier $^{\mathrm{a}}$ \\ Yvon C. Chagnon ${ }^{\mathrm{a}, \mathrm{c}}$ Marc-André Roy ${ }^{\mathrm{a}, \mathrm{c}}$ Michel Maziade ${ }^{\mathrm{a}, \mathrm{c}}$ \\ ${ }^{a}$ Centre de recherche de l'institut universitaire en santé mentale de Québec, ${ }^{b}$ Département de médecine sociale et \\ préventive, and 'Département de psychiatrie et neurosciences, Université Laval, Québec, Qué., Canada
}

\section{Key Words}

Bipolar disorder · Parametric linkage analysis $\cdot$ Pedigree

analysis $\cdot$ Phenotype model $\cdot$ Schizophrenia

\begin{abstract}
Objective: To increase power to detect modifier loci conferring susceptibility to specific phenotypes such as disease diagnoses which are part of a broader disorder spectrum by jointly modeling a modifier and a broad susceptibility gene and to identify modifier loci conferring specific susceptibility to schizophrenia (SZ) or to bipolar disorder (BP) using the approach. Methods: We implemented a two-locus linkage analysis model where a gene 1 genotype increases the risk of a broad phenotype and a gene 2 genotype modifies the expression of gene 1 by conferring susceptibility to a specific phenotype. Results: Compared to a single-locus analysis within the broad phenotype, the proposed approach had greater power to detect the modifier gene 2 (0.96 vs. 0.54 under a simulation scenario including heterogeneity). In a sample of 12 mixed SZ and BP Eastern Quebec kindreds, D8S1110 at 8p22 showed the strongest evidence of linkage to a gene determining a specific phenotype (SZ or BP) among subjects susceptible to major psychosis because of putative genes at 10p13 (D10S245, conditional maximized LOD $(\mathrm{cMOD})=4.20, \mathrm{p}=0.0003)$ and 3q21-q23 (D3S2418, $\mathrm{cMOD}=$
\end{abstract}

4.09, $p=0.0005)$. Conclusion: The proposed strategy is useful to detect modifier loci conferring susceptibility to a specific phenotype within a broader phenotype.

Copyright $\odot 2012$ S. Karger AG, Basel

\section{Introduction}

In complex disorders, specific phenotypes such as disease diagnoses are often part of a broader syndrome or disorder spectrum, and both a specific phenotype of interest and other forms of the broader phenotype can be observed in different members of the same family. For instance, schizophrenia (SZ) and bipolar disorder (BP) are two forms of the spectrum of major psychosis (MP), which also includes schizo-affective disorder $[1,2]$.

We posit that a mechanism may trigger the onset of a specific phenotype in patients who would already carry a genetic predisposition to the broad phenotype. For highly heritable diseases, this mechanism may be a gene modifying the effect of the genes predisposing to the broad phenotype. For example, de Pontual et al. [3] provided evidence that mutations in the RET gene conferred susceptibility to Hirschsprung disease among carriers of mutations in a Bardet-Biedl syndrome gene.

\section{KARGER}

Fax +4161306 1234

E-Mail karger@karger.ch

www.karger.com
(C) 2012 S. Karger AG, Basel

$0001-5652 / 12 / 0734-0195 \$ 38.00 / 0$

Accessible online at:

www.karger.com/hhe
Alexandre Bureau

Médecine sociale et préventive, 1050 rue de la Médecine, room 2457 Québec, QC G1V 0A6 (Canada)

Tel. +1 418656 2131, ext. 3342, E-Mail alexandre.bureau@fmed.ulaval.ca 
The term 'modifier gene' is commonly used to refer to genes leading to a quantitative or qualitative difference in a disease phenotype [4]. While it has been mostly used to designate genes modifying the effect of a known mutation causing a Mendelian trait, the term also applies in the absence of well-characterized genes predisposing to what we call a broad phenotype. In that context, the search for modifier loci has traditionally been conducted by performing a single-locus analysis within the broad phenotype, i.e. a model in which subjects with the specific phenotype are considered affected, subjects with the broad phenotype but without the specific phenotype are considered unaffected, and finally subjects without the broad phenotype are considered unknown [4]. We are proposing instead a two-locus genetic modeling of a modifier gene where a gene 1 genotype increases the risk of the broad phenotype and a gene 2 genotype modifies the expression of gene 1 by conferring susceptibility to a specific phenotype. The modifier gene for the specific phenotype is added to usual one-locus parametric models for the broad phenotype by extending our previously proposed strategy for linkage analysis under epistasis, which allows the epistatic effects to act only in a subset of families from a sample through a family selection step [5]. This paper presents evidence that the two-locus model can improve the power to detect the modifier locus by linkage analysis over the analysis within the broad phenotype described above.

The paper is organized as follows: first, after defining the proposed model space, we explain how we insert it into our two-locus linkage analysis strategy. Second, we evaluate the strategy by simulation. Third, we illustrate its application in real data from Eastern Quebec kindreds affected by both SZ and BP to identify loci conferring susceptibility to $\mathrm{SZ}$ or to BP specifically by modifying the effect of MP susceptibility genes.

\section{Methods}

\section{Definition of Model Space and Notation}

We previously defined a space of two-locus epistatic models for a disease (specific phenotype) where the two-locus genotypes formed by combining the genotypes $G_{1}$ and $G_{2}$ of two genes are divided into two groups, susceptible and non-susceptible, and susceptible genotypes include disease susceptibility (DS) alleles at both loci [5]. The disease penetrance equals $\beta_{0}$ for non-susceptible genotypes and $\beta_{0}+\beta_{1}$ for susceptible ones, with the constraints $\beta_{0}, \beta_{1} \geq 0$ and $0<\beta_{0}+\beta_{1} \leq 1$. In the joint modeling of a modifier and a broad susceptibility gene, an epistatic model is used for the specific phenotype $S$, and additional penetrance parameters $\gamma_{0}$ and $\gamma_{1}$ are introduced to expand the model to the broad phe- notype $B$. Formally, we denote by $X_{j}=X\left(G_{j}\right)$ the coding of the effect of the genotype $G_{j}$ at the $j$-th locus. For instance, for a dominant locus, $X_{j}=1$ if $G_{j}=A / a$ or $A / A$, and $X_{j}=0$ if $G_{j}=a / a$. Then, the penetrance functions take the form:

$$
\begin{aligned}
& f_{S}\left(G_{1}, G_{2}\right)=\beta_{0}+\beta_{1} X_{1} X_{2} \\
& f_{B}\left(G_{1}, G_{2}\right)=\beta_{0}+\gamma_{0}+\gamma_{1} X_{1}+\left(\beta_{1}-\gamma_{1}\right)_{+} X_{1} X_{2}
\end{aligned}
$$

assuming that $X_{1}$ and $X_{2}$ are binary, and where $\left(\beta_{1}-\gamma_{1}\right)_{+}$is the maximum of $\beta_{1}-\gamma_{1}$ and 0 . The parameter $\gamma_{0}$ represents, for nonsusceptible genotypes, the risk of phenotypes other than the specific one being considered but remaining within the broad phenotype, and $\gamma_{1}$ represents the effect of gene 1 on the risk of the broad phenotype in the absence of a risk genotype at gene 2 . The last term of $f_{B}$ reflects the fact that carriers of risk genotypes in both genes who do not develop the specific phenotype are nonetheless at risk for the broad phenotype. The constraints $\gamma_{0}, \gamma_{1} \geq 0,0<$ $\gamma_{0}+\beta_{0}+\gamma_{1} \leq 1$ and $0<\gamma_{0}+\beta_{0}+\beta_{1} \leq 1$ insure that penetrances lie between 0 and 1 . Online supplementary figure 1 (for all online suppl. material, see www.karger.com/doi/10.1159/000341392) shows a graphical representation of a penetrance model with $\beta_{1}>$ $\gamma_{1}$. The probabilities for the three mutually exclusive phenotypic categories (specific phenotype, other phenotypes within the broad phenotype, and unaffected subjects) are presented in tabular format in table 1 for a dominant-dominant model in the subclass of models where $\beta_{1}>\gamma_{1}$.

In the sub-class of models where $\beta_{1} \leq \gamma_{1}$, the probability of being unaffected, $1-f_{B}$, is independent of the gene 2 genotype. As a result, the contrast between subjects with the specific phenotype and subjects with other phenotypes within the broad phenotype provides most of the information on linkage to gene 2. The analysis of markers linked to gene 2 based on such two-locus models defined above is, however, not mathematically equivalent to the analysis within the broad phenotype under a one-locus model, and consideration of the two loci simultaneously can still provide power gains, as we show in our simulations reported below.

\section{Coding the Three Phenotypic Categories in Linkage Analysis} Software

Currently available linkage analysis software is designed for dichotomous affected/unaffected phenotypes and does not allow multiple phenotypic categories. One can, however, call all subjects with the broad phenotype 'affected' and the others 'unaffected' and code our trichotomous model with three penetrance matrices: $f_{S}$ for subjects with the specific phenotype, $f_{B}-f_{S}$ for subjects with phenotypes other than the specific phenotype but within the broad phenotype, and $f_{B}$ for unaffecteds (i.e. the matrix of probabilities of being unaffected is $1-f_{B}$ ). The appropriate matrix is assigned to each subject using 'liability classes'.

\section{Analysis Strategy}

The steps of a modifier gene analysis under the proposed twolocus modifier gene model and a single-locus analysis within the broad phenotype are listed in table 2. The main steps of the twolocus analysis are detailed below. We focus in this work on analyzing one highly polymorphic marker at each locus, but the statistics generalize to multipoint analyses of biallelic single nucleotide polymorphisms (SNPs), with the recombination fractions replaced by positions on marker maps in two unlinked regions as 
Table 1. Probabilities for the three phenotypic categories under the proposed penetrance model for linkage analysis for a dominantdominant model

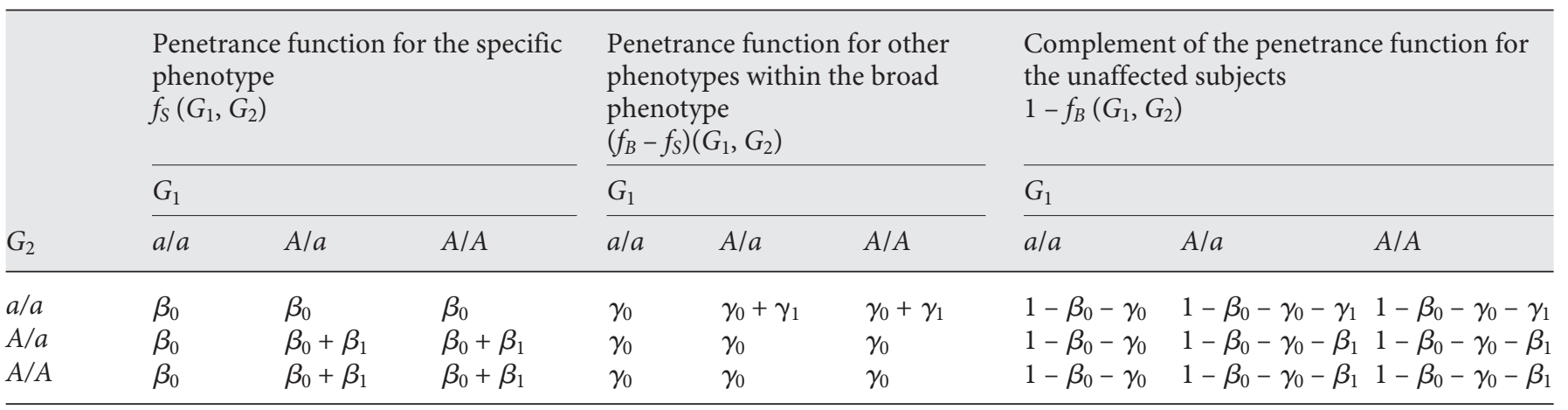

$G_{1}=$ Genotype at locus $1 ; G_{2}=$ genotype at locus 2 . The penetrances shown correspond to the case where $\beta_{1} \geq \gamma_{1}$.

Table 2. Steps of the strategies for detecting a modifier gene by linkage analysis using two-locus and single-locus analyses ${ }^{\mathrm{a}}$

\begin{tabular}{|c|c|c|c|}
\hline \multicolumn{2}{|c|}{ Steps } & \multirow{2}{*}{$\begin{array}{l}\text { Two-locus modifier gene approach } \\
\text { Compare } Z^{2 L} \text { and } Z^{1 L}\end{array}$} & \multirow{2}{*}{$\begin{array}{l}\text { Single-locus analysis } \\
\text { within broad phenotype } \\
\text { NA }\end{array}$} \\
\hline $1 \mathrm{~A}$ & Pre-selection of candidate markers (optional) & & \\
\hline $1 \mathrm{~B}$ & Marker pairing & $\begin{array}{l}\text { Make a list of pairs of conditioning candidate } \\
\text { markers }\end{array}$ & NA \\
\hline 3 & $\begin{array}{l}\text { Address heterogeneity } \\
\text { - by selecting family subset } \\
\text { or }\end{array}$ & $\begin{array}{l}Z^{\text {SLICED+ }} \text { or } Z^{C O N D+} \\
Z^{H-S L I C E D} \text { or } Z^{H-C O N D}\end{array}$ & $\begin{array}{l}Z_{w}^{L+} \\
Z^{H-1 L}\end{array}$ \\
\hline
\end{tabular}

a See text and table 3 for definition of the notation.

discussed in Bureau et al. [5]. Affected as well as unaffected pedigree members contribute to the phenotypic and genotypic data. The various functions of log-likelihoods of the data that can be computed under various models are listed in table 3. The evidence for linkage of both markers to their respective disease locus is measured by the two-locus two-marker LOD score:

$$
Z^{2 L}\left(\theta_{1}, \theta_{2}, k\right)=l^{2 L}\left(\theta_{1}, \theta_{2}, k\right)-l^{2 L}(1 / 2,1 / 2, k) .
$$

\section{Pre-Selection of Markers and Marker Pairing (Step 1)}

We assume that conditioning markers are markers already identified in a single-locus analysis of the broad phenotype. The search for modifier loci is then conditional on one or a few of these previously linked markers. Candidate markers can include all other available markers on the genome or in candidate regions, or be pre-selected using the Step 1 criteria of Bureau et al. [5], namely (a) that the maximum of the two-locus one-marker LOD score $Z^{2 L}\left(1 / 2, \theta_{2}, k\right)$ exceeds the maximum of $Z^{1 L}\left(\theta_{2}, h\right)=l^{1 L}\left(\theta_{2}, h\right)-$ $l^{1 L}(1 / 2, h)$ by a pre-specified margin or (b) exceeds a pre-specified threshold. The difference with Bureau et al. [5] is that here, $Z^{2 L}\left(1 / 2, \theta_{2}, k\right)$ is computed using the penetrance model for the specific and broad phenotypes defined by equations (1) and (2). Selected candidate markers are then paired to the conditioning markers.

\section{Computation of Conditional LOD Scores (Step 2)}

The linkage signal at the locus linked to the hypothesized modifier gene is assessed by conditioning on the linkage information at the locus of the gene predisposing to the broad phenotype. Conditioning on locus 1 implies subtracting $l^{2 L}\left(\theta_{1}, 1 / 2, k\right)$ from $l^{2 L}\left(\theta_{1}, \theta_{2}, k\right)$. We previously defined the conditional LOD score at locus 2 as

$$
Z^{C O N D}\left(\theta_{2}, k\right)=l^{2 L}\left(\hat{\theta}_{1}(k), \theta_{2}, k\right)-l^{2 L}\left(\breve{\theta}_{1}(k), 1 / 2, k\right),
$$


Table 3. Notation for functions of log-likelihoods

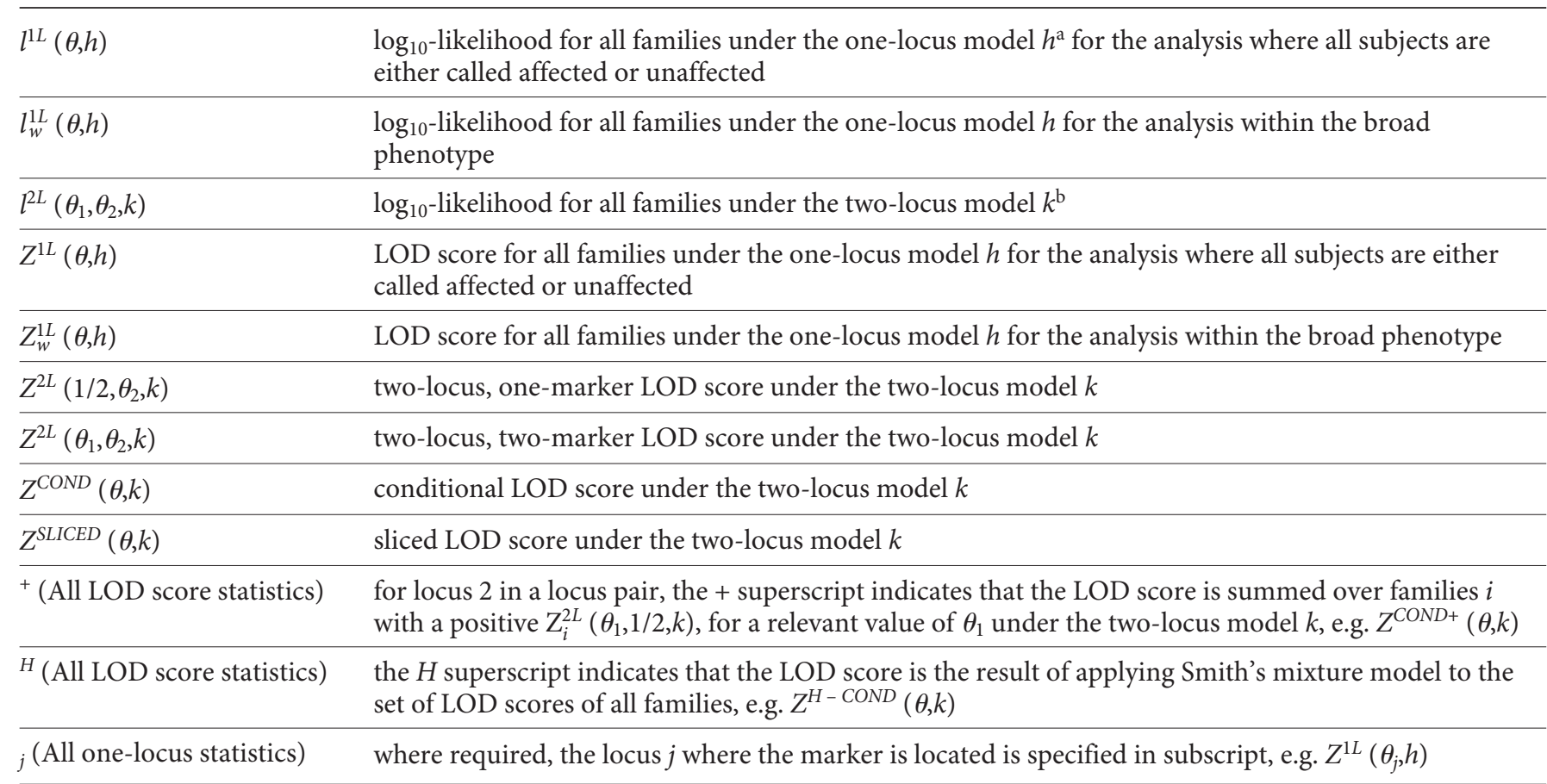

${ }^{a} h$ represents a vector $\left(\beta_{0}, \beta_{1}, p_{1}, \delta_{1}\right)$ of penetrance parameters $\beta_{0}, \beta_{1}$, allele frequency $p_{1}$ and dominance indicator $\delta_{1}(0=$ recessive/ $1=$ dominant) for a one-locus model.

${ }^{\mathrm{b}} k$ represents a vector $\left(\beta_{0}, \beta_{1}, \gamma_{1}, \gamma_{2}, p_{1}, p_{2}, \delta_{1}, \delta_{2}\right)$ of penetrance parameters $\beta_{0}, \beta_{1}, \gamma_{1}, \gamma_{2}$ allele frequencies $p_{1}, p_{2}$ and dominance indicators $\delta_{1}, \delta_{2}$ for a two-locus modifier model.

where $\hat{\theta}_{1}(k)$ maximizes $Z^{2 L}\left(\theta_{1}, \theta_{2}\right)$ at the current value of $\theta_{2}$ and $\breve{\theta}_{1}(k)$ maximizes $Z^{2 L}\left(\theta_{1}, 1 / 2, k\right)$ under model $k$ [5]. Other authors [6-10] used the 'sliced' LOD score:

$$
Z^{\text {SLICED }}\left(\theta_{2}, k\right)=l^{2 L}\left(\hat{\theta}_{1}(k), \theta_{2}, k\right)-l^{2 L}\left(\hat{\theta}_{1}(k), 1 / 2, k\right) .
$$

Selection of Families Linked to the Conditioning Marker to Address Heterogeneity (Step 3)

Only a fraction of the families in a sample may be linked to the pair of loci being modeled because of genetic heterogeneity. Conditioning the analysis of one locus on the other offers a way to deal with heterogeneity by selecting families showing evidence for linkage to the conditioning locus, for instance selecting family $i$ if its LOD score $Z_{i}^{2 L}\left(\hat{\theta}_{1}(k), 1 / 2, k\right)>0$. The resulting conditional and sliced LOD scores are denoted $Z^{C O N D+}$ and $Z^{\text {SLICED+ }}$, respectively. We have previously shown that this strategy is powerful and does not inflate the conditional LOD score nor the sliced LOD score for the candidate locus for a fixed genetic model [5]. The one-locus LOD score $Z_{w}^{1 L}$ of the analysis within the broad phenotype at locus 2 described in the introduction can also be computed on the same sets of families with positive LOD score at a conditioning locus, and we denote the score obtained by summing families with $Z_{i}^{2 L}\left(\breve{\theta}_{1}(k), 1 / 2, k\right)>0$ as $Z_{w}^{1 L+}$. An alternative to deal with genetic heterogeneity is to use the mixture model of Smith [11] to compute a heterogeneity LOD (HLOD) for any of the analyses.
Maximization of the LOD Score over a Space of Models

As we did before [5], LOD scores can be maximized over the parameters of the penetrance functions to obtain a maximized LOD or MOD score [12], preventing parameter misspecification while constraining the prevalence of the specific and broad phenotypes to their population value. Models with constrained prevalence are more realistic, and fewer models are needed to thoroughly cover constrained model spaces in the maximization over a grid of models, reducing the computational burden. The prevalence $\pi_{S}$ of the specific phenotype in an unselected population is given by

$$
\pi_{S}=\beta_{0}+\beta_{1} P\left[X_{1} X_{2}\right],
$$

where $P\left[X_{1} X_{2}\right]$ is the prevalence of the genotypes such that $X_{1}$ and $X_{2}$ are both equal to 1 , again assuming $X_{1}$ and $X_{2}$ are binary variables. Similarly, the prevalence $\pi_{B}$ of the broad phenotype is given by

$$
\pi_{B}=\beta_{0}+\gamma_{0}+\gamma_{1}\left(P\left[X_{1}\right]\right)+\left(\beta_{1}-\gamma_{1}\right)_{+} P\left[X_{1} X_{2}\right] .
$$

Under the assumptions of Hardy-Weinberg and linkage equilibrium between the two disease loci, the genotype frequencies are determined by the frequencies of the DS alleles at the two loci $p_{1}$ and $p_{2}$. For instance, $P\left[X_{1} X_{2}\right]=\left(1-\left(1-p_{1}\right)^{2}\right)\left(1-\left(1-p_{2}\right)^{2}\right)$ for a dominant-dominant model. With a set of values for $\gamma_{0}, \gamma_{1}, \beta_{0}$ and $\beta_{1}$ we obtain $p_{1}$ and $p_{2}$ by substituting $P\left[X_{1} X_{2}\right]$ and $P\left[X_{1}\right]$ in equa- 
tions (7) and (8) by their expressions in terms of $p_{1}$ and $p_{2}$, and then numerically solving the two resulting equations. In this work, we considered values of 0 and $\pi_{S} / 2$ for $\beta_{0}, 0$ and $\left(\pi_{B}-\pi_{S}\right) / 2$ for $\gamma_{0}$, and a range from 0.1 to 0.9 for both $\beta_{1}$ and $\gamma_{1}$.

\section{Software}

We used Superlink $[13,14]$ to perform linkage computations, the only currently available program capable of executing twolocus two-marker computations in large pedigrees. We wrote scripts in Perl and the S language for the R environment to set up Superlink input files for the analysis of pairs of regions containing either only one or multiple markers under sets of models, and to process output files. These scripts are available upon request.

\section{Simulation Study}

\section{Simulation Study Setup}

We used the same simulated three-generation pedigree samples with the same specific phenotype and genotype data as in Bureau et al. [5]. The penetrance matrices and allele frequencies for the three simulated models are given in online supplementary table 1 . The parameter values were chosen to obtain prevalences in unascertained populations of 1 and 5.3\% for the specific and broad phenotypes, respectively. Under the first two scenarios, the modifying effect of gene 2 is to determine whether a subject is at risk of the specific phenotype or another form of the broad phenotype. The first two scenarios differ principally in the penetrance of the broad phenotype: under scenario 1, it goes from $52 \%$ with a gene 1 risk genotype alone to $89 \%$ with the combination of risk genotypes at genes 1 and 2; under scenario 2, it is unchanged by the gene 2 genotype, remaining $89 \%$. Our ascertainment criterion for scenarios 1 and 2 required at least a sib pair and one cousin with the specific phenotype. We further required that at least one of the two pivot individuals be unaffected by the specific phenotype to force a non-Mendelian transmission pattern for the specific phenotype as we did before [5]. The above condition was satisfied in $53 \%$ of simulated families, and data sets were also generated without it. Scenario 3 is outside the modifier gene model space to assess the behavior of the statistics under model misspecification: genotypes at gene 2 confer risk to the specific phenotype irrespective of the genotype at gene 1; genotypes at the unlinked gene 1 confer risk for other forms of the broad phenotype in subjects that are not carriers of risk genotypes at gene 2 , the opposite of modifier gene models. Taking the broad phenotype as a whole, it appears to be caused by the presence of DS alleles at either of two loci (genetic heterogeneity). For this third scenario, we required at least one subject with the specific pheno-

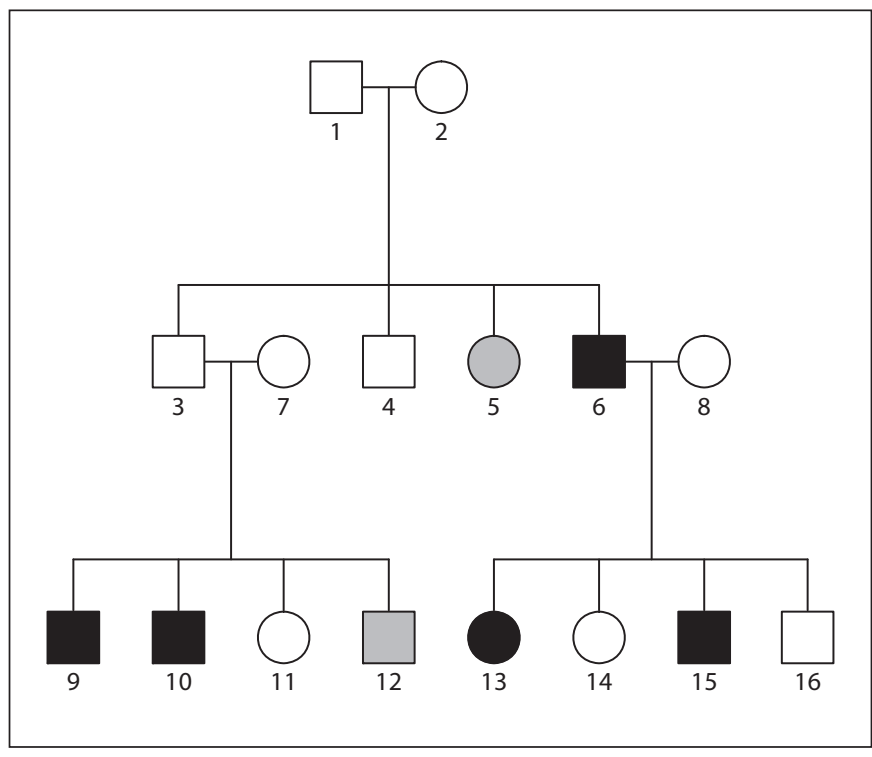

Fig. 1. Pedigree structure used in the simulation study, with a representative distribution of the phenotypes. Black filled symbols represent the subjects affected by the specific phenotype, gray symbols represent the subjects affected by the broad phenotype without the specific phenotype, and empty symbols represent unaffected subjects.

type and at least one subject with the other form of the broad phenotype in each family. The presence of both forms of the broad phenotype insured that both genes were present in most families. The phenotypes of a representative family are shown in figure 1 . Descriptive statistics on the number of subjects with each phenotype are displayed in online supplementary table 2 .

Figure 2 depicts the simulation study setup. Simulated family samples were either homogeneously generated from one of the three models or an equal mixture of families from a two-locus model and families from a singlelocus dominant model with specific and broad phenotype penetrances chosen so that their respective prevalences approximately equal those in the families linked to loci 1 and 2. In addition to family samples with markers linked to both disease loci, we also simulated samples of 10 families under the null hypothesis where the candidate marker is unlinked $\left(\theta_{2}=1 / 2\right)$ to obtain the null distribution of our statistics.

We simulated a marker on top of the disease locus on each chromosome, each with four equifrequent alleles, resulting in heterozygosity of $75 \%$. All phenotype and marker genotype data were simulated using the computer package Simla [15]. 
Table 4. Quantiles of null distribution of linkage statistics in simulated families (with 95\% CI)

\begin{tabular}{|c|c|c|c|c|c|c|}
\hline \multirow[t]{2}{*}{ Statistic } & \multirow[t]{2}{*}{ Model } & \multicolumn{5}{|l|}{ Significance level } \\
\hline & & 0.05 & 0.01 & 0.005 & 0.0017 & 0.001 \\
\hline \multirow[t]{2}{*}{$Z^{2 L}\left(\theta_{1}, 1 / 2\right)$} & fixed $^{b}$ & $0.62(0.60,0.65)$ & $1.21(1.17,1.27)$ & $1.46(1.39,1.54)$ & $1.87(1.74,2.05)$ & $2.12(1.95,2.31)$ \\
\hline & best $^{\mathrm{c}}$ & $1.42(1.40,1.45)$ & $2.14(2.10,2.20)$ & $2.42(2.37,2.51)$ & $2.90(2.71,3.10)$ & $3.14(2.97,3.30)$ \\
\hline$Z^{C O N D}$ & best $^{c}$ & $1.34(1.31,1.36)$ & $2.03(1.97,2.09)$ & $2.28(2.21,2.34)$ & $2.71(2.58,2.83)$ & $2.98(2.72,3.16)$ \\
\hline \multirow[t]{2}{*}{$Z^{S L I C E D}$} & fixed $^{b}$ & $0.62(0.61,0.65)$ & $1.20(1.15,1.25)$ & $1.43(1.38,1.51)$ & $1.83(1.72,1.96)$ & $2.09(1.92,2.35)$ \\
\hline & best $^{\mathrm{c}}$ & $1.34(1.31,1.36)$ & $2.03(1.97,2.08)$ & $2.28(2.21,2.34)$ & $2.72(2.58,2.83)$ & $2.98(2.76,3.12)$ \\
\hline \multirow[t]{2}{*}{$Z^{C O N D+}$} & fixed $^{b}$ & $0.62(0.60,0.64)$ & $1.21(1.16,1.26)$ & $1.48(1.40,1.53)$ & $1.90(1.76,2.05)$ & $2.11(1.93,2.24)$ \\
\hline & best $^{\mathrm{c}}$ & $1.49(1.47,1.52)$ & $2.16(2.13,2.22)$ & $2.44(2.34,2.54)$ & $2.87(2.77,3.05)$ & $3.11(2.98,3.23)$ \\
\hline
\end{tabular}

${ }^{\mathrm{a}}$ Quantiles of the one-sided $\chi^{2}{ }_{(1)}$ distribution multiplied by $2 \log 10 .{ }^{\mathrm{b}}$ LOD scores are obtained by fixing one model, defined by the parameters $\beta_{0}=\pi_{S} / 2=0.005, \gamma_{0}=\left(\pi_{B}-\pi_{S}\right) / 2=0.0215, \beta_{1}=0.9$ and $\gamma_{1}=0.5{ }^{\mathrm{c}}{ }^{\mathrm{LO}}$ LO scores are maximized over the family of epistatic models considered in this paper with the prevalences of the specific and broad phenotypes set to $\pi_{S}=0.01$ and $\pi_{B}=0.053$.

Fig. 2. Simulation setup.

\begin{tabular}{|c|c|c|c|c|}
\hline \multicolumn{2}{|c|}{$\begin{array}{c}\text { Scenarios } 1 \text { and } 2 \\
2 \text { DS loci } \\
\text { (chromosomes } 1 \text { and 2) }\end{array}$} & \multicolumn{2}{|c|}{$\begin{array}{c}1 \text { DS locus } \\
\text { (chromosome 3) }\end{array}$} & $\begin{array}{c}\text { Scenario } 3 \\
2 \text { DS loci } \\
\text { (chromosomes } 1 \text { and 2) }\end{array}$ \\
\hline $\begin{array}{l}10 \text { families } \\
\text { momogeneous } \\
\text { sample }\end{array}$ & 20 families & $\begin{array}{l}20 \text { families } \\
\text { geneous } \\
\text { ingle-locus } \\
40 \text { families) }\end{array}$ & $\begin{array}{l}10 \text { families } \\
\text { Single-locus } \\
\text { sample (for null } \\
\text { distribution) }\end{array}$ & $\begin{array}{c}20 \text { families } \\
\text { Homogeneous } \\
\text { non-modifier model } \\
\text { sample }\end{array}$ \\
\hline 100 replicates & $100 \mathrm{re}$ & plicates & 20,000 replicates & 100 replicates \\
\hline
\end{tabular}

For analyses conditioning on locus 1, power was calculated in two ways: (1) assuming that the analysis is performed irrespective of the one-locus linkage to locus 2 and (2) assuming that the analysis is performed only when the Step 1A criteria are satisfied (pre-select).

\section{Simulation Results}

The estimated quantiles of the null distribution of the statistics considered in this paper are presented in table 4 . For a fixed genetic model, the quantiles of the distribution of the statistics are close to those from the theoretical $\chi^{2}$ distribution. Maximization over the models shifted the distributions to the right: at the significance level of 0.0017 recommended for suggestive linkage in a genome scan [16], the quantile is inflated by $0.9-1.0$ compared to using a fixed model. These null distributions were used to compute the $\mathrm{p}$ values of the statistics in simulations with markers linked to two loci. All results reported here involve markers located on top of a disease gene. We analyzed the simulated data following the two approaches described in table 2: the two-locus modifier gene approach, maximizing over the grid of parameter values 
Table 5. Signal at modifier locus (locus 2) using joint modeling of a specific and a broad phenotype under simulation scenario 1

\begin{tabular}{|c|c|c|c|c|c|c|c|c|c|}
\hline & \multirow[t]{2}{*}{ Statistic ${ }^{\mathrm{a}}$} & \multicolumn{4}{|c|}{$\begin{array}{l}\text { Two genes only } \\
\text { (100 replicate samples of } 10 \text { families) }\end{array}$} & \multicolumn{4}{|c|}{$\begin{array}{l}\text { Two genes plus heterogeneity } \\
\text { (100 replicate samples of } 40 \text { families) }\end{array}$} \\
\hline & & mean $(\mathrm{SD})$ & mean $\theta(\mathrm{SD})$ & power $^{\mathrm{b}}$ & $\begin{array}{l}\text { power }^{\mathrm{b}} \\
\text { (pre-select) }\end{array}$ & mean $(\mathrm{SD})$ & mean $\theta(\mathrm{SD})$ & power $^{\mathrm{b}}$ & $\begin{array}{l}\text { power }^{\mathrm{b}} \\
\text { (pre-select) }\end{array}$ \\
\hline \multicolumn{10}{|l|}{ All families } \\
\hline \multirow[t]{2}{*}{ One-locus analyses } & LOD within broad $\left(Z_{w}^{1 L}\right)$ & $2.48(1.45)$ & $0.11(0.07)$ & 0.48 & NA & $1.81(1.48)$ & $0.25(0.08)$ & 0.26 & NA \\
\hline & HLOD within broad $\left(Z_{w}^{H-1 L}\right)$ & $2.69(1.40)$ & $0.07(0.07)$ & 0.52 & NA & $2.61(1.53)$ & $0.08(0.08)$ & 0.44 & NA \\
\hline \multirow{2}{*}{ Two-locus analyses } & Conditional HLOD $\left(Z^{H-C O N D}\right)$ & $4.36(1.46)$ & $0.01(0.04)$ & 0.87 & 0.87 & $4.16(1.59)$ & $0.03(0.05)$ & 0.83 & 0.85 \\
\hline & Sliced HLOD $\left(Z^{H-S L I C E D}\right)$ & $4.36(1.46)$ & $0.01(0.04)$ & 0.87 & 0.88 & $4.11(1.58)$ & $0.03(0.05)$ & 0.82 & 0.85 \\
\hline \multicolumn{10}{|l|}{ Selected families } \\
\hline One-locus analyses & LOD within broad $\left(Z_{w}^{1 L+}\right)^{\mathrm{c}}$ & $2.51(1.52)$ & $0.09(0.07)$ & 0.46 & NA & $2.86(1.69)$ & $0.16(0.07)$ & 0.54 & NA \\
\hline Two-locus analyses & Conditional LOD $\left(Z^{\mathrm{COND}+}\right)$ & $4.14(1.49)$ & $0.02(0.04)$ & 0.78 & 0.80 & $5.65(1.89)$ & $0.07(0.06)$ & 0.96 & 0.96 \\
\hline
\end{tabular}

presented in the Methods section, and a single-locus analysis within subjects with the broad phenotype, again maximizing over the model space.

We selected replicates where locus 1 was strongly linked to the broad phenotype $\left(Z^{1 L}\left(\theta_{1}\right)>3\right)$. Table 5 and online supplementary table 3 present the linkage signals at the modifier locus (gene 2) under scenarios 1 and 2, respectively. We observe that the two-locus modifier gene approach provides an important gain in power to detect the modifier locus 2 over the single-locus approach within subjects with the broad phenotype under scenario 1 (table 5, two-locus vs. one-locus statistics), where the penetrance of the broad phenotype depends on genes 1 and 2 . The power gains are more modest under scenario 2 (online suppl. table 3, two-locus vs. one-locus statistics), where the penetrance of the broad phenotype depends on gene 1 only, but are nonetheless unlikely due to random variation in the simulations. For instance, in the simulation under homogeneity the power of $Z^{C O N D}$ is significantly greater than the power of $Z_{w}^{1 L}(0.97$ vs. 0.84 , McNemar test $\left.\mathrm{p}=2.4 \times 10^{-4}\right)$. We examined the models which maximized $Z^{C O N D}$ in that simulation and found that $\gamma_{1}=\beta_{1}$ in 12 out of 13 discrepant replicates where $Z^{C O N D}$ rejected the null, but the LOD within the broad phenotype did not. The two-locus analysis therefore provides greater signals at gene 2 even under models where the penetrance of the broad phenotype depends on gene 1 only.

Detection of Phenotype Modifier Genes Using Two-Locus Linkage Analysis
Under scenarios 1 and 2, the strategy of summing the families with a positive LOD at locus 1 was more powerful than applying a mixture model (table 5 and online suppl. table 3 , selected families vs. HLOD statistics). In the single-locus analysis of locus 2 , the summation over families positive at locus $1\left(Z_{w}^{1 L+}\right)$ actually becomes a twolocus analysis, while the mixture model remains a singlelocus analysis. All the above observations under scenarios 1 and 2 also apply to simulations without the condition that families must have a pivot unaffected by the specific phenotype (see online suppl. table 4 for results under scenario 1$)$.

Under scenario 3, where the underlying genetic model is outside the modifier gene model space, a standard onelocus analysis where subjects with the specific phenotype are called affected and all others are called unaffected $\left(Z^{1 L}\right.$ and $\left.Z^{H-1 L}\right)$ is also reported. The modifier gene analysis of the full sample (two-locus statistics) maintained a power similar to the standard analysis and slightly higher than the analysis within the broad phenotype, whether or not a mixture model was applied (online suppl. table 5). Since, under this scenario, families with a strong linkage at locus 1 tended to have few carriers of gene 2 DS alleles, selecting families linked to locus 1 tended to decrease the signal and power at locus 2, contrary to scenarios 1 and 2.

In the presence of a specific and broad phenotype, applying the epistatic analysis proposed in Bureau et al. [5] 


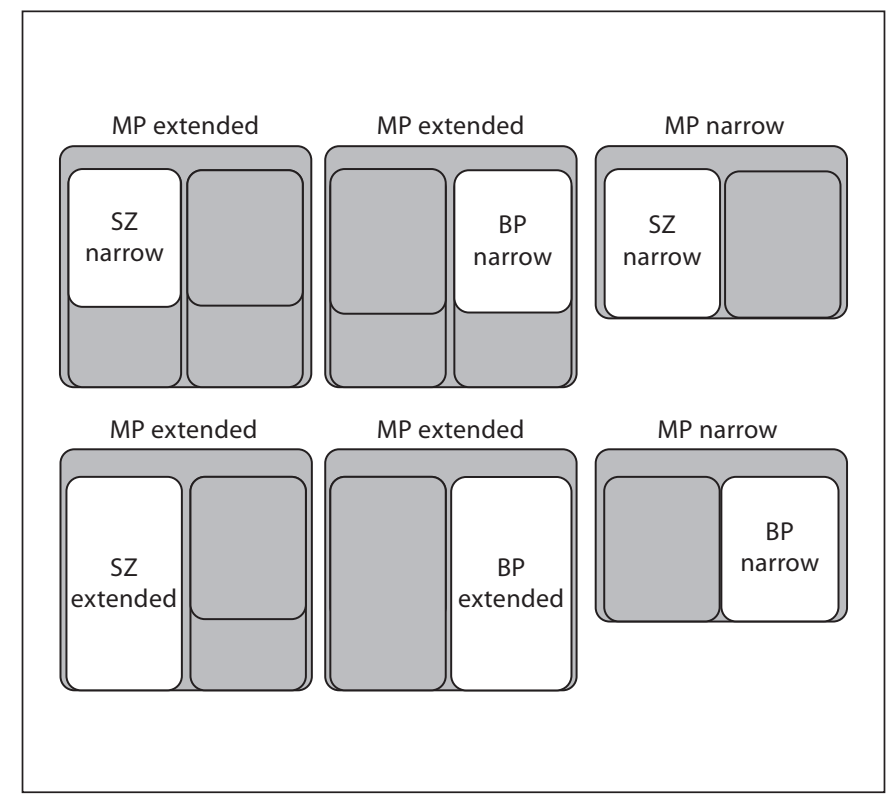

Fig. 3. Diagram of the phenotypic category in the Eastern Quebec SZ and BP kindred study.

to the specific phenotype ignores the information provided by the broad phenotype. A comparison between table 3 of Bureau et al. [5] and the results under scenarios 1 and 2 where the same epistatic model was used to simulate the specific phenotype shows that the modifier approach is more powerful than all analyses considering only the specific phenotype.

\section{Translation of Proposed Methods to Our Sample of SZ and BP Kindreds}

Our group and others have extensively documented commonalities in mechanisms across SZ and BP as indicated by their familial co-aggregation $[1,17]$, neurocognitive and neurobiological endophenotypes $[18,19]$ and several genetic linkage or association signals [2, 20-22]. In addition to genes conferring susceptibility to the broad spectrum of MP spanning SZ and BP, we hypothesize that other genes may modify the effect of these genes and lead to the specific expression of either SZ or BP. To the best of our knowledge, the present analysis is the first to simultaneously model a specific and a broad susceptibility gene in this context.

\section{Sample Description}

We tested our modifier gene hypothesis in our sample of SZ and BP kindreds from Eastern Quebec. Among the 48 kindreds, we already reported [20] that 12 of the 48 kindreds presented both SZ and BP cases in comparison to the other families that were predominantly affected by either SZ or BP, i.e. no more than $15 \%$ of patients were affected by the other disorder in a family. We restricted our primary analysis to that subset of 12 mixed families because of their greater informativeness to detect modifier loci. The subsample comprised 291 family members of whom 215 provided a DNA sample.

\section{Phenotype Description}

A best-estimate lifetime DSM-IV diagnosis was made with stringent procedures outlined in previous reports [23-25]. Ascertainment, diagnostic methods and genotyping procedures for microsatellite markers were detailed elsewhere [2, 23-26]. Although we conceptualize $\mathrm{SZ}$ and BP as specific phenotypes, the boundaries of these disorders are not well defined. We have therefore considered two definitions of each disorder: one narrow and one extended (fig. 3). Note that the extended definitions correspond to what we termed the 'broad' definitions in previous publications $[2,20,26]$. We use 'extended' here to avoid confusion with the concept of broad phenotype described in this article, which in this particular application corresponds to the MP phenotype. For SZ, we used a narrow definition restricted to $\mathrm{SZ}(\mathrm{n}=39)$ and an extended definition comprising SZ plus schizophreniform disorder and schizotypal personality $(n=42)$. For BP, the narrow definition was restricted to BP I $(n=24)$ and the extended definition included BP I, BP II and recurrent major depression ( $\mathrm{n}=$ 38). The broad MP phenotype narrow $(\mathrm{n}=73)$ and extended $(n=90)$ definitions mirrored those of BP and SZ. Online supplementary table 6 reports the values for the prevalence of the various phenotypes that we used to constrain the specific and broad phenotype prevalences in the analysis.

\section{Pre-Selection of Markers and Marker Pairing}

The pre-selection had three stages. First, we focused our analysis on markers located in the 10 regions (2q12$\mathrm{q} 22,3 \mathrm{q} 21-\mathrm{q} 23,6 \mathrm{p} 22,10 \mathrm{p} 13,12 \mathrm{q} 23,13 \mathrm{q} 13-14,15 \mathrm{q} 11$, $15 \mathrm{q} 26,16 \mathrm{p} 13$ and $18 \mathrm{q} 12-\mathrm{q} 21)$ in which we previously reported genome-wide significant or suggestive linkage signals to either SZ, BP or MP in a subsample of kindreds [2], plus the SCZD6 locus on 8p where the signal was only slightly weaker. Second, for conditioning, we selected 7 
markers in 5 regions as linked to the extended definition of the MP phenotype with $Z^{1 L}\left(\theta_{1}, h\right)>1.5$. One of these markers, D6S334, was also linked to the narrow definition of the MP phenotype. Third, we selected 14 markers as candidates for linkage to a modifier gene because the maximum of $Z^{2 L}\left(1 / 2, \theta_{2}, k\right)$ exceeded the maximum of $Z^{1 L}\left(\theta_{2}, h\right)$ by more than 0.8 or was $>2.75$, for either $S Z$ or $\mathrm{BP}$, in their narrow or extended definition (online suppl. table 7).

We then formed 105 conditioning marker-candidate marker pairs to apply our proposed approach. To form a pair, the definition of the MP phenotype in the twolocus model used for the candidate marker had to be the same as the one used for the conditioning marker. For instance, D18S1145 satisfied the candidate marker criterion in an analysis involving the narrow version of the MP phenotype; it was therefore paired only with the sole conditioning marker selected based on the narrow version of the MP phenotype (D6S334). Consequently, not all candidate markers were tested with all conditioning markers. A Bonferroni correction was applied for the number of regions containing conditioning markers to control the type I error. The 14 candidate markers were also tested in a single-locus analysis where SZ or BP patients were considered affected, other psychotic patients were considered unaffected and nonpsychotic subjects were treated as unknown (withinMP analysis). We simulated 50,000 replicates of our family sample under the null hypothesis where the candidate marker is unlinked $\left(\theta_{2}=1 / 2\right)$ to obtain the null distribution of the statistics.

\section{Computing Time}

We timed the computations on a single $2.8 \mathrm{GHz}$ Intel Xeon X5660 processor of a HP DL380G6 computer with $72 \mathrm{~Gb}$ of RAM. In a sample of 10 simulated families (fig. 1), the average time required to perform the analysis of a locus pair under a fixed two-locus model was $3.43 \mathrm{~s}$ with one four-allele marker at each locus and $15.75 \mathrm{~min}$ in multipoint analyses with 11 biallelic SNPs at each locus. For maximization over a grid of 93 models, the total time was $5.3 \mathrm{~min}$ using one marker and $24.4 \mathrm{~h}$ using 11 SNPs. In the sample of 12 Eastern Quebec kindreds, the computing time averaged over several multi-allele marker pairs was 24 min under a fixed two-locus model and $37 \mathrm{~h}$ with 93 models. Multipoint analysis in the Eastern Quebec kindreds was limited to two biallelic markers by our computer RAM. The 11 SNP analyses could therefore not be performed.

Detection of Phenotype Modifier Genes

Using Two-Locus Linkage Analysis

\section{Results}

Table 6 shows the results of the analysis for the marker pairs where at least one statistic had a p value $<0.0005$. Marker D8S1110 at 8p22 shows the strongest evidence of linkage to a gene modifying the action of a MP susceptibility gene. The specific phenotype giving the strongest signal varies depending on the analysis: BP (narrow) when conditioning on 10p13 (marker D10S245) and SZ (extended) when conditioning on 3q21-q23 (marker D3S2418) in subsets of families linked to each of these two conditioning loci (table 6). The within-MP analysis $\left(Z_{w}^{1 L}\left(\theta_{2}\right)\right.$ also yielded a signal of similar statistical significance at D8S1110 when using SZ (extended) as specific phenotype (table 6). The latter signal was higher in the subset of families linked to D3S2418 (3.89) than in all families (3.29), but its statistical significance was lower after Bonferroni correction for subsetting using multiple conditioning markers. The phenotype switching from one analysis to another is unsurprising, given that, for a diallelic modifier gene in psychotic patients, if one genotype predisposes to SZ, carriers of the other genotype have in most instances a BP diagnosis, and vice versa, since most psychotic patients have either SZ or BP (fig. 3). Online supplementary figure 2 shows the genotypes of the members of the family with the greatest contribution to the BP signal at D8S1110 when conditioning on D10S245, where the modifying effect of a gene linked to D8S1110 can be observed through the marker genotypes. It is noteworthy that, for the narrow BP-specific phenotype when conditioning on D10S245, the model which maximized the signal at D8S1110 implies a greater penetrance for the specific than the other phenotype $\left(\beta_{1}>\gamma_{1}\right)$ and gives a more significant signal than the within-MP analysis while, for the extended SZ phenotype when conditioning on D3S2418, the model which maximized the signal at D8S1110 had the same penetrance for the specific and other phenotypes $\left(\beta_{1}=\gamma_{1}\right)$ and a $p$ value similar to that of the within-MP analysis (online suppl. table 8). This is consistent with the power of the withinMP analysis being closer to that of the two-locus analysis when $\beta_{1}=\gamma_{1}$.

When we further investigated the above findings by repeating the analysis of the D10S245-D8S1110 and D3S2418-D8S1110 marker pairs using alternative phenotype definitions that were not retained by our selection procedure, the conditional LOD score $Z^{C O N D+}$ was reduced in most instances, with the exception of the analysis using the narrow version of the BP and MP phenotypes $\left(Z^{\mathrm{COND+}}=4.45\right)$. 
Table 6. Conditional linkage signals under epistatic models of SZ and BP as specific phenotypes of MP in families from Eastern Quebec, with and without selection of families linked to the conditioning marker

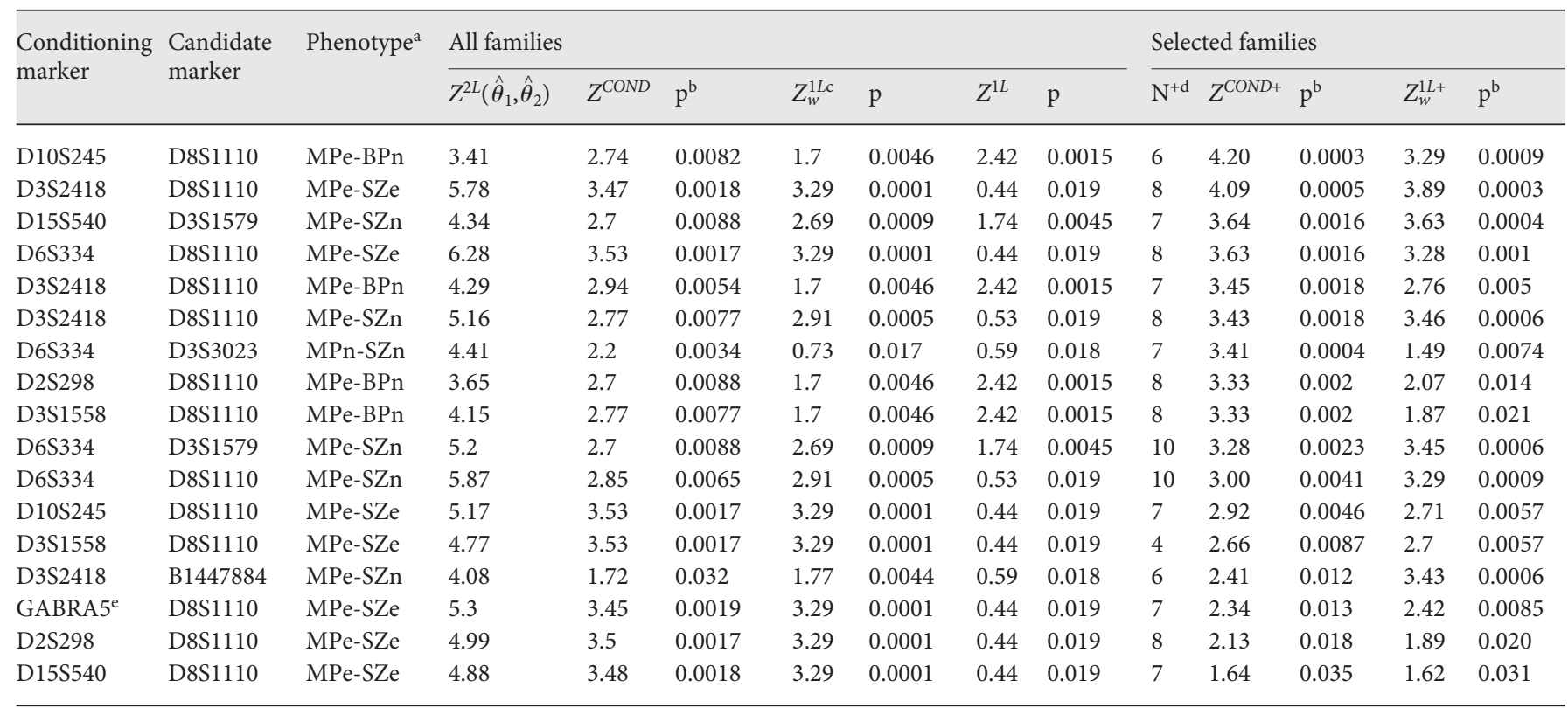

a 'MP', 'BP' and 'SZ' stand for 'Major Psychosis', 'Bipolar Disorder' and 'Schizophrenia'; the suffix ' $n$ ' or 'e' is added to specify whether the narrow or extended definition of the disorder is considered. ${ }^{b} \mathrm{p}$ values for conditional LOD scores have been multiplied by the number of regions containing markers that were used for conditioning (same phenotype and outside the region of the candidate marker) to control the type I error. ${ }^{\mathrm{c}}$ Single-locus analysis within subjects with the broad phenotype. ${ }^{\mathrm{d}}$ Number of informative families with $Z_{i}^{2 L}\left(\breve{\theta}_{1}(k), 1 / 2, k\right)>0 .{ }^{\mathrm{e}}$ This marker is located in the $15 \mathrm{q}$ region.

To further mine the data, we also added the homogeneous kindreds to the mixed kindreds in two different analyses: (1) the 21 BP families, for a total of 33 families, to extend the analyses involving BP as specific phenotype, and (2) the $15 \mathrm{SZ}$ families, for a total of 27 families, to extend the analyses involving SZ as specific phenotype. For the BP analysis, $Z^{C O N D+}$ jumped to 6.34 and the within-MP LOD score to 5.76 in the subset of 15 families showing positive linkage to D10S245. In all 33 families, the within-MP $Z_{w}^{1 L}\left(\theta_{2}\right)$ increased to 3.76. However, the overall linkage evidence between D10S245 and MP in the 33 families using either the LOD or $\operatorname{HLOD} Z^{1 L}\left(\theta_{1}\right)$ fell below our preset threshold of 1.5 . For the SZ analysis, $Z^{C O N D+}$ was slightly reduced to 4.01 and the within-MP LOD score to 3.56 in the 10 families showing positive linkage to D3S2418. In all 27 families, the within-MP $Z_{w}^{1 L}\left(\theta_{2}\right)$ fell to 0.75 .

A limitation of our procedure for the pre-selection of candidate markers is that LOD scores are computed using all families, the LODs being therefore subject to the negative impact of genetic heterogeneity. In our sample, we had to set the threshold for $Z^{1 L}\left(\theta_{1}, h\right)$ as low as 1.5 to find markers linked to the MP phenotype. Using the HLOD did not select any more markers. We then had the idea to select subsets of families to deal with heterogeneity when evaluating linkage to the MP phenotype using criteria inspired from the Step 1A criteria. Namely, for a given pair of markers, we sum the $Z_{i}^{1 L}\left(\theta_{1}, h\right)$ over the families $i$ which have $Z_{i}^{2 L}\left(1 / 2, \hat{\theta}_{2},(k), k\right)>0$. When marker 2 in the pair is unlinked to any modifier gene, this procedure amounts to selecting a random subset of families for testing linkage to marker 1, which does not introduce bias. When marker 2 is linked to a modifier locus, the procedure selects markers where the families linked to marker 2 are also linked to marker 1 . These families are those most likely to contribute to $Z^{C O N D+}$ and $Z^{\text {SLICED+ }}$ for marker 2. For D10S245, $Z^{1 L}\left(\theta_{1}, h\right)$ went from 1.57 in all 12 families to 2.89 in the 8 families with $Z_{i}^{2 L}\left(1 / 2, \hat{\theta}_{2},(k), k\right)>$ 0 at D8S1110. Applying this approach to all marker pairs, we obtained 9 pairs with $Z^{1 L}\left(\theta_{1}, h\right)>2$ that were not previously selected using all families and the 1.5 threshold. Among these, we note that the marker pair with D8S1110 linked to a gene conferring risk for BP (narrow) as specific phenotype, and D10S183 linked to a susceptibility gene for the extended version of the MP phenotype yields a $Z^{C O N D+}$ of 4.90 (uncorrected $\mathrm{p}=2.0 \times 10^{-5}$ ) in 
a subset of 6 families, compared to 2.7 (uncorrected $\mathrm{p}=$ 0.0017 ) in all families. D10S183 is located $8.06 \mathrm{Mb}$ from D10S245.

\section{Discussion}

We proposed a model accounting for the action of two genes, one of them conferring susceptibility to a broad phenotype such as MP and the other acting over the first to facilitate the expression of a specific phenotype, while allowing for genetic heterogeneity between families. This model may be useful in the comprehension of the demonstrated commonality between SZ and BP, and of the familial co-aggregation of both disorders in a large proportion of affected families [1, 17].

Our simultaneous modeling of a specific and broad phenotype in the same families involves penetrance functions used in parametric linkage analysis of categorical traits. A simple procedure involving liability classes enabled the implementation of the method with standard parametric linkage analysis software designed for dichotomous phenotypes. Nonparametric linkage methods, which are essentially affected-only methods, do not allow the same flexibility in modeling multiple phenotypic categories. The problem of model misspecification, the downside of parametric linkage analysis, is attenuated by maximization of LOD scores over a large space of genetic models.

When translating our model into actual family data, the analysis of SZ and $\mathrm{BP}$ highlighted the $8 \mathrm{p} 11$ region around D8S1110 as likely to contain a gene modifying MP susceptibility to confer either the SZ- or BP-specific phenotype. In this case, the single-locus LOD score of 3.29 from the analysis of SZ within the MP phenotype had approximately the same $\mathrm{p}$ value as the 4.20 LOD score conditional on D10S245. Despite the lack of a gain in statistical significance, the two-locus analysis had the advantage of pointing to $3 \mathrm{q} 21-\mathrm{q} 23$ and $10 \mathrm{p} 13$ as regions which may contain a gene whose phenotypic expression is modified by a gene near D8S1110.

In the situation considered in this work (an epistatic interaction between two loci causing a specific phenotype within a broader phenotype), the joint modeling of a modifier and a broad susceptibility gene under the proposed model is more powerful than our previously proposed two-locus epistatic analysis [5] applied to the specific phenotype, as revealed in the simulation study and the analysis of marker pairs involving D8S1110. Indeed, the epistatic analysis yielded a LOD of 1.01 for BP (nar-

Detection of Phenotype Modifier Genes

Using Two-Locus Linkage Analysis row) at D8S1110 conditional on D10S245 on a subset of 8 families and a LOD of 2.06 for SZ (extended) at D8S1110 conditional on D3S2418 on a subset of 3 families. The consideration of both specific phenotypes and a broad MP phenotype was therefore required to detect linkage to D8S1110 in the mixed families. However, the analysis of a specific phenotype under an epistatic model remains relevant when no appropriate broad phenotype exists or when no linkage to the broad phenotype is detected at a conditioning locus. We illustrate that point with the linkage signal for the narrow BP phenotype at a marker on 8 p11 close to D8S1110, conditional on a marker on 16p13 reported in Bureau et al. [5]. The absence of linkage to MP at $16 \mathrm{p} 13$ in the subset of mixed SZ-BP kindreds prevented conditioning on markers in that region in the current analysis (we also note that in our previously reported linkage to BP at $16 \mathrm{p} 13$ in a single-locus affected-only analysis [26], only 2 families among the 6 in the D10S245D8S1110 selected subset and only 1 family among the 8 in the D3S2418-D8S1110 selected subset had a NPL pair $>1.0$ ). Furthermore, the subset of families contributing to the previously reported 8 p11 signal did not overlap with the D10S245-D8S1110 and D3S2418-D8S1110 selected family subsets. It is unknown at this point whether the two 8p11 signals are the result of the same gene interacting with different other genes in different subsets of families, or whether two distinct genes in the $8 \mathrm{p} 11$ region are producing distinct signals. The limited overlap with families responsible for other linkage signals is an indication of the genetic heterogeneity in our sample, which was resolved here by the selection integrated into our approach (Step 3).

A simpler alternative to our proposed model to detect modifier loci is to perform a single-locus analysis for the specific phenotype, calling 'unaffected' only those subjects with other forms of the broad phenotype (analysis within the broad phenotype). Our simulation study revealed that using the proposed model had greater power than that simpler alternative when conditioning was limited to a single locus linked to the broad phenotype and the underlying genetic model was within the space of modifier models. The power gain depended on the underlying model, with maximal gain when the penetrance of the broad phenotype was function of the genotype of both genes in the model. Simulations covering a broader range of generating models would be needed to thoroughly evaluate all factors influencing the power gain. When the underlying model was outside the modifier model space, the modifier gene approach maintained a power similar to that of a standard single-locus analysis 
due to a certain flexibility to adapt to the data within the modifier model space. The approach was designed for the purpose of locus detection, not model identification, and the above results illustrate that attempting to interpret the underlying genetic model based on LOD scores would be hazardous.

In the presence of multiple linkage signals to the broad phenotype, performing a search for modifier loci conditional on every signal increases the odds of finding pairs of loci where the model fits well, but requires a multiple testing correction for the number of regions containing conditioning markers, contrary to the single-locus analysis within the broad phenotype. That correction may reduce the power of the two-locus approach below that of the single-locus analysis for loci with a form of modifying effect under which the single-locus analysis is powerful.
Nonetheless, the ability of the two-locus analysis to point pairs of loci where one modifies the effect of the other, as in the SZ and BP analysis, is an advantage that leads us to recommend the two-locus approach over the single-locus analysis within the broad phenotype whenever a broad phenotype has been linked to genomic loci and specific phenotypes are present.

\section{Acknowledgements}

This work was funded by the Canadian Institutes of Health Research (CIHR, grant MOP-220168). A.B. is supported by a research fellowship from the Fonds de recherche du Québec - Santé. The Eastern Quebec Kindred Study is funded by CIHR (grants MT-12854 and MOP-74430) and by a Canada Research Chair (No. 950-200810) in the genetics of neuropsychiatric disorders of which M.M. is the Chair.

\section{References}

1 Van Snellenberg JX, de Candia T: Meta-analytic evidence for familial coaggregation of schizophrenia and bipolar disorder. Arch Gen Psychiatry 2009;66:748-755.

$\checkmark 2$ Maziade M, Roy MA, Chagnon YC, Cliche D, Fournier JP, Montgrain N, Dion C, Lavallee JC, Garneau Y, Gingras N, Nicole L, Pires A, Ponton AM, Potvin A, Wallot H, Merette C: Shared and specific susceptibility loci for schizophrenia and bipolar disorder: a dense genome scan in Eastern Quebec families. Mol Psychiatry 2005;10:486-499.

3 de Pontual L, Zaghloul NA, Thomas S, Davis EE, McGaughey DM, Dollfus H, Baumann C, Bessling SL, Babarit C, Pelet A, Gascue C, Beales P, Munnich A, Lyonnet S, Etchevers H, Attie-Bitach T, Badano JL, McCallion AS, Katsanis N, Amiel J: Epistasis between RET and BBS mutations modulates enteric innervation and causes syndromic Hirschsprung disease. Proc Natl Acad Sci USA 2009;106: 13921-13926.

4 Houlston RS, Tomlinson IP: Modifier genes in humans: strategies for identification. Eur J Hum Genet 1998;6:80-88.

$\checkmark 5$ Bureau A, Merette C, Croteau J, Fournier A, Chagnon YC, Roy MA, Maziade M: A new strategy for linkage analysis under epistasis taking into account genetic heterogeneity. Hum Hered 2009;68:231-242.

-6 Cordell HJ, Todd JA, Bennett ST, Kawaguchi Y, Farrall M: Two-locus maximum lod score analysis of a multifactorial trait: joint consideration of IDDM2 and IDDM4 with IDDM1 in type 1 diabetes. Am J Hum Genet 1995;57:920-934.
Cordell HJ, Wedig GC, Jacobs KB, Elston RC: Multilocus linkage tests based on affected relative pairs. Am J Hum Genet 2000;66: 1273-1286.

8 Schork NJ, Boehnke M, Terwilliger JD, Ott J: Two-trait-locus linkage analysis: a powerful strategy for mapping complex genetic traits. Am J Hum Genet 1993;53:1127-1136.

-9 Sung YJ, Thompson EA, Wijsman EM: MCMC-based linkage analysis for complex traits on general pedigrees: multipoint analysis with a two-locus model and a polygenic component. Genet Epidemiol 2007;31:103-114.

10 Sung YJ, Wijsman EM: Accounting for epistasis in linkage analysis of general pedigrees. Hum Hered 2007;63:144-152.

11 Smith CA: Testing for heterogeneity of recombination fraction values in human genetics. Ann Hum Genet 1963;27:175-182.

12 Clerget-Darpoux F, Bonaiti-Pellie C, Hochez J: Effects of misspecifying genetic parameters in lod score analysis. Biometrics 1986;42: 393-399.

13 Fishelson M, Geiger D: Exact genetic linkage computations for general pedigrees. Bioinformatics 2002;18(suppl 1):S189-S198.

14 Fishelson M, Geiger D: Optimizing exact genetic linkage computations. J Comput Biol 2004;11:263-275.

15 Schmidt M, Hauser ER, Martin ER, Schmidt $S$ : Extension of the SIMLA package for generating pedigrees with complex inheritance patterns: environmental covariates, genegene and gene-environment interaction. Stat Appl Genet Mol Biol 2005;4:article 15.

16 Lander E, Kruglyak L: Genetic dissection of complex traits: guidelines for interpreting and reporting linkage results. Nat Genet 1995;11:241-247.
17 Lichtenstein P, Yip BH, Bjork C, Pawitan Y, Cannon TD, Sullivan PF, Hultman CM: Common genetic determinants of schizophrenia and bipolar disorder in Swedish families: a population-based study. Lancet 2009;373:234-239.

18 Hébert M, Gagne AM, Paradis ME, Jomphe V, Roy MA, Merette C, Maziade M: Retinal response to light in young nonaffected offspring at high genetic risk of neuropsychiatric brain disorders. Biol Psychiatry 2010;67: 270-274.

19 Maziade M, Rouleau N, Merette C, Cellard C, Battaglia M, Marino C, Jomphe V, Gilbert E, Achim A, Bouchard RH, Paccalet T, Paradis ME, Roy MA: Verbal and visual memory impairments among young offspring and healthy adult relatives of patients with schizophrenia and bipolar disorder: selective generational patterns indicate different developmental trajectories. Schizophr Bull 2011;37:1218-1228.

20 Maziade M, Chagnon YC, Roy MA, Bureau A, Fournier A, Merette C: Chromosome 13q13-q14 locus overlaps mood and psychotic disorders: the relevance for redefining phenotype. Eur J Hum Genet 2009; 17:10341042.

21 Purcell SM, Wray NR, Stone JL, Visscher PM, O'Donovan MC, Sullivan PF, Sklar P: Common polygenic variation contributes to risk of schizophrenia and bipolar disorder. Nature 2009;460:748-752. 
22 Williams HJ, Craddock N, Russo G, Hamshere ML, Moskvina V, Dwyer S, Smith RL, Green E, Grozeva D, Holmans P, Owen MJ, O'Donovan MC: Most genome-wide significant susceptibility loci for schizophrenia and bipolar disorder reported to date cross-traditional diagnostic boundaries. Hum Mol Genet 2011;20:387-391.

23 Maziade M, Raymond V, Cliche D, Fournier JP, Caron C, Garneau Y, Nicole L, Marcotte P, Couture C, Simard C, Boivin R, Rodrigue C, Boutin P, De Braekeleer M, Martinez M, Mérette C: Linkage results on 11q21-22 in
Eastern Quebec pedigrees densely affected by schizophrenia. Am J Med Genet 1995;60: 522-528.

24 Maziade M, Roy MA, Fournier JP, Cliche D, Mérette C, Caron C, Garneau Y, Montgrain $\mathrm{N}$, Shriqui C, Dion C, Nicole L, Potvin A, Lavallée JC, Pirès A, Raymond V: Reliability of best-estimate diagnosis in genetic linkage studies of major psychoses: results from the Québec pedigree studies. Am J Psychiatry 1992;149:1674-1686.

25 Roy MA, Lanctôt G, Mérette C, Cliche D, Fournier JP, Boutin P, Rodrigue C, Charron
L, Turgeon M, Hamel M, Montgrain N, Nicole L, Pirès A, Wallot $\mathrm{H}$, Ponton AM, Garneau Y, Dion C, Lavallée JC, Potvin A, Szatmari P, Maziade M: Clinical and methodological factors related to reliability of the best-estimate diagnostic procedure. Am J Psychiatry 1997;154:1726-1733.

26 Merette C, Roy MA, Bureau A, Fournier A, Emond C, Cliche D, Jomphe V, Chagnon YC, Maziade M: Replication of linkage with bipolar disorder on chromosome $16 \mathrm{p}$ in the Eastern Quebec population. Am J Med Genet B Neuropsychiatr Genet 2008;147B:737-744. 